\title{
Adaptive meiotic drive in selfing populations with heterozygote advantage
}

\author{
Evgeny Brud
}

\begin{abstract}
Department of Ecology and Evolution, Stony Brook University, Stony Brook, NY, 11790
Current address: Department of Biology, University of Pennsylvania, Philadelphia, PA, 19104
\end{abstract} email: ebrud@sas.upenn.edu

\section{Abstract}

The egalitarian allotment of gametes to each allele at a locus (Mendel's law of segregation) is a near-universal phenomenon characterizing inheritance in sexual populations. As exceptions to Mendel's law are known to occur, one can investigate why non-Mendelian segregation is not more common using modifier theory. Earlier work assuming sex-independent modifier effects in a random mating population with heterozygote advantage concluded that equal segregation is stable over long-term evolution. Subsequent investigation, however, demonstrated that the stability of the Mendelian scheme disappears when sex-specific modifier effects are allowed. Here I derive invasion conditions favoring the repeal of Mendelian law in mixed and obligate selfing populations. Oppositely-directed segregation distortion in the production of male and female gametes is selected for in the presence of overdominant fitness. The conditions are less restrictive than under panmixia in that strong selection can occur even without differential viability of reciprocal heterozygotes (i.e. in the absence of parent-of-origin effects at the overdominant fitness locus). Generalized equilibria are derived for full selfing.

Keywords: mating system, segregation distortion, overdominance, modifier theory

\section{Introduction}

Despite a growing number of examples where the rule of Mendelian segregation is violated (e.g. Fishman and Saunders 2008, Didion et al. 2016, Nuckolls et al. 2017), exceptions to fair meiosis in the form of meiotic drivers are thought to be relatively uncommon (Crow 1991). Among the exceptions, many cases of meiotic drive may be transient in that they involve the spread of an initially rare segregation distorter that quickly rises all the way to fixation. Drivers of this sort are consistent with the observation of widespread adherence to the Mendelian scheme, since fixation entails the loss of manifestation of the drive phenotype. Other cases of meiotic drive may involve homozygous fitness costs of sufficient magnitude to allow for the initial increase of a driver but not its fixation, and so give rise to 
a condition of persistent variation for segregation distortion as is found in mice (e.g. thaplotypes; Dunn 1957), Drosophila (e.g. SD-haplotypes; Sandler, Hiraizumi, and Sandler 1959), the chromosomal knobs of maize (Rhoades 1942), and various cases of sex chromosome drive (e.g. Gershenson 1928), among a number of other examples; often such drivers carry a number of fitness costs in both heterozygous and homozygous genotypes (reviewed in Burt and Trivers 2006). But even these cases are unstable in the long run,

42 since an intermediate frequency of a selfish driver will select for suppressors throughout

43 the genome, and so the existence of segregation distortion at some locus is regularly

44 destabilized by adaptive countermeasures aimed at restoring the Mendelian order.

45 Considerations such as these seem to argue that Mendelian ratios ought to be widespread

46 (in accord with observation) and evolutionarily stable. However, Úbeda and Haig (2005)

47 proved the existence of conditions in which rare modifiers are expected to invade a

48 resident population by virtue of promoting meiotic drive at unlinked autosomal loci subject

49 to fitness variation. In the present work, I demonstrate that the conditions for evolving a

50 stable phenotype of meiotic drive are even less restrictive when self-fertilization is

51 included in the model.

52 The general evolutionary causes of autosomal adherence to Mendelian segregation has

53 received sporadic attention in the theoretical population genetics literature since it was

54 first modeled in the 1970s (Hartl 1975; Liberman 1976, 1990; Thomson and Feldman

55 1976; Liberman and Feldman 1980, 1982; Lloyd 1984; Eshel 1985; Úbeda and Haig 2005,

56 Brandvain and Coop 2015). One branch of these efforts assumes a random mating

57 population with a focal locus subject to di-allelic variation and heterozygote advantage, in 
which the alleles have evolved to a stable equilibrium. A second locus is assumed to be variable for alleles that modify the segregation ratio at the focal fitness locus. The key question is: what are the conditions favoring the spread of a rare modifier? Early models investigated sex-independent variables and parameters. Notable results include Liberman (1976), who found that in a population fixed for a resident phenotype of Mendelian segregation, rare modifiers could spread under broad conditions: any rate of recombination less than one-half can result in the invasion of a drive-enhancer (i.e. a modifier that imposes or increases a deviation from equal segregation) given that it is sufficiently strong, with tight linkage being especially conducive to invasions. Eshel (1985) showed that in the case of free recombination, the only kinds of modifiers which can invade are those that reduce the intensity of drive; enhancers are uniformly selected against. These results recall the metaphor of Leigh (1977) who likened the genome to "a parliament of genes" which enforces behavior consistent with the common good against "'cabals of a few' conspiring for their 'selfish profit'” (p. 4543). Modifiers with sufficient linkage to a distorting locus can form a selfish cabal, but the presumably greater number of unlinked modifiers act to police outlaw behavior.

An unsatisfying aspect of the work mentioned above is the assumption of sex-independent effects. If a sex-differentiated population is assumed, and if accordingly modifiers have sexspecific effects on male and female segregation, then an analysis of modifier invasion demonstrates that unlinked genes should evolve to subvert Mendelian ratios after all (Úbeda and Haig 2005). The benefit in doing so owes to the assumption that a modifier acts on a locus exhibiting heterozygote advantage. Oppositely-directed segregation schemes, in which an allele has a segregation advantage in one sex but a segregation disadvantage in 
81 the other sex, are selected for as a mechanism of producing a super-Mendelian proportion

82 of fit heterozygous offspring, and thus evolve to reduce the genetic load caused by the

83 production of unfit homozygous progeny (segregation load). This possibility was absent in

84 the sex-independent models. On the assumption of symmetric overdominance, in which

85 parent-of-origin effects on fitness are absent (i.e. "Aa" and "aA" genotypes have identical

86 fitnesses, where the order of the alleles distinguishes parental origin), rare modifiers of the

87 segregation scheme are selected to increase at a slow arithmetic rate. Asymmetric scheme of "all-and-none segregation", in which ratios are maximally distorted in male and female meiosis, but in opposite directions (see also Úbeda and Haig 2004).

The importance of the sex-specific results with respect to biological phenomena is unclear given that asymmetric overdominance in fitness is likely a rare class of balanced polymorphism. Such a fitness scheme involves the simultaneous realization of heterozygote advantage and parent-of-origin effects (e.g. genomic imprinting), examples of which are lacking. Another factor constraining the spread of adaptive drive is that with respect to symmetric overdominance, the repeal of the Mendelian process is associated 
104

105

106

107

108

109

110

111

112

113

114

115

116

\section{$117 \quad$ Model}

118

119

120

121

122

123

124

reproduction (e.g. male fertility reduction). Nevertheless, their results reveal that the reasons for the ubiquity of Mendelian segregation are more obscure than is generally appreciated (Úbeda 2006).

The impact of inbreeding on the invasion of unlinked modifiers of drive in the context of heterosis has not previously been investigated, and it is reasonable to suspect that the mating system will have a qualitative impact on the evolutionary dynamics of adaptive meiotic drive. Here I investigate a model that extends the conditions under which Mendelian segregation is selected against to the case of partially and fully selfing populations. Invasion conditions are derived for unlinked modifiers that impose a mutant segregation scheme in the context of symmetric overdominance in fitness. Likewise, I assess the long-term stability of all-and-none segregation to unlinked modifiers that alter the ratios closer to equality. In addition, I derive generalized equilibrium genotype frequencies for the case of full selfing.

Assuming a monoecious population with sex-differentiated meiosis, I examine a two-locus, two-allele discrete-generation deterministic model with an autosomal fitness locus, $A$, and an unlinked autosomal modifier locus, $B$. The fitness scheme is $\mathrm{W}_{\mathrm{Aa}}=1>\mathrm{W}_{\mathrm{AA}}, \mathrm{W}_{\mathrm{aa}} \geq 0$. In heterozygotes, the resident segregation ratio of $A: a$ is equal to $1-k_{m 0}: k_{m 0}$ in sperm and 1$k_{f 0}: k_{f 0}$ in eggs of wild-type BB individuals, and in the mutant genotypes $\mathrm{Bb}$ or bb, the ratio is $1-k_{m}: k_{m}$ in sperm and $1-k_{f}: k_{f}$ in eggs. Part of the population (1-S) are the offspring resulting from outcrossing, assuming random union of gametes. The remainder $(S)$ owe to 
125

126

127

128

129

130

131

132

133

134

135

136

137

138

139

140

141

142

143

144

145

diploid selfing. With 10 possible two-locus diploid genotypes, the dynamical equations for the genotypic proportions are given by:

$$
p_{i j}^{\prime}=(1-S)\left(x_{i} y_{j}+\left(1-\delta_{i j}\right) x_{j} y_{i}\right)+\left(\bar{W}^{-1} S\right)\left(\mathbf{p} \mathbf{T}_{i j}\right)
$$

, which is valid for $i, j=1,2,3,4$ and such that $i j$ and $j i$ are not distinguishable. $p_{i j}^{\prime}$ is the next-generation frequency of the genotype composed of haplotypes $i$ and $j$ (the labels are $\mathrm{AB}=1, \mathrm{Ab}=2, \mathrm{aB}=3, \mathrm{ab}=4)$. The $x_{i}$ and $y_{j}$ terms are the male and female gametic frequencies, respectively, subsequent to zygotic selection of diploids, and are given by $x_{i}=$ $\bar{W}^{-1}\left(\mathbf{p} \mathbf{U}_{\boldsymbol{i}}\right)$ and $y_{j}=\bar{W}^{-1}\left(\mathbf{p} \mathbf{V}_{\boldsymbol{j}}\right)$, where $\bar{W}$ is the mean population fitness, equal to $\sum \sum p_{i j} \mathrm{~W}_{i j}$. $\mathbf{p}$ is a row vector of genotype frequencies prior to zygotic selection, and such that $\sum \sum p_{i j}=1 . \mathrm{W}_{i j}$ is the zygotic fitness of genotype $i j$ and is equal to $\mathrm{W}_{\mathrm{AA}}$ for $i j=11,12,22$ and is equal to $\mathrm{W}_{\mathrm{aa}}$ for $i j=33,34,44$; all other subscripts correspond to heterozygotes associated with a fitness of $1 . \mathbf{U}_{\boldsymbol{i}}$ and $\mathbf{V}_{\boldsymbol{j}}$ are the $i^{\text {th }}$ and $j^{\text {th }}$ column vectors from the $10 \times 4$ matrices ( $\mathbf{U}$ and $\mathbf{V}$ ) governing transmission and selection in outcrossers. $\delta_{i j}$ is the Kronecker delta. $\mathbf{T}_{\boldsymbol{i}}$ is a column vector corresponding to the production of genotype $i j$ from the 10x10 matrix (T) describing transmission and selection in selfers. $\mathbf{U}, \mathbf{V}$, and $\mathbf{T}$ are presented in the appendix.

\section{Results}

External stability of a Mendelian partial selfing population to adaptive meiotic drive

Assuming a population is initially fixed for the Mendelian-favoring BB genotypes, variation at $A$ will be determined by the forces of selection against homozygotes and the rate of selfing. Equilibrium properties for single-locus systems of selfing and overdominant fitness 
164

have been investigated previously (Hayman 1953, Workman and Jain 1966). The state space for a single-locus partial selfing population with two alleles is fully described by the allele frequency (q) and the inbreeding coefficient (F). One can formulate a model in which $x=p^{2}+F p q ; y=2 p q(1-F) ; z=q^{2}+F p q$, where the frequency of $A$ is $p=1-q$, and the frequencies of $\mathrm{AA}, \mathrm{Aa}$, and aa are $x, y$, and $z$, respectively. (Aa and aA are not distinguished). The influences of selection, selfing, and Mendelian segregation are such that in the next generation genotypic frequencies are

$$
\begin{aligned}
& x^{\prime}=\bar{W}^{-1}(S)\left(x \mathrm{~W}_{\mathrm{AA}}+\frac{y}{4}\right)+(1-S)\left(p_{a}\right)^{2} \\
& y^{\prime}=\bar{W}^{-1}(S)\left(\frac{y}{2}\right)+(1-S)\left(2 p_{a} q_{a}\right) \\
& z^{\prime}=\bar{W}^{-1}(S)\left(z \mathrm{~W}_{\mathrm{aa}}+\frac{y}{4}\right)+(1-S)\left(q_{a}\right)^{2}
\end{aligned}
$$

, where $p_{a}=\bar{W}^{-1}\left(\frac{y}{2}+x \mathrm{~W}_{\mathrm{AA}}\right), q_{a}=\bar{W}^{-1}\left(\frac{y}{2}+z \mathrm{~W}_{\mathrm{aa}}\right)$ and $\bar{W}=x \mathrm{~W}_{\mathrm{AA}}+y+z \mathrm{~W}_{\mathrm{aa}}$ are respectively the frequency of $A$ and $a$ alleles among outcrossers after selection $\left(p_{a}, q_{a}\right)$ and $\bar{W}$ is the mean fitness of the population. Since $q^{\prime}=z^{\prime}+\frac{y^{\prime}}{2}$ and $F^{\prime}=1-\frac{y^{\prime}}{2 p^{\prime} q^{\prime}}$ are the frequency of the $a$ allele and the inbreeding coefficient in the next generation, setting the change in $q$ and $F$ over successive generations equal to zero yields a pair of equilibria in $\hat{q}$ and $\widehat{F}$, only one of which is biologically feasible in the interior of allele frequency space (i.e. such that both $\hat{q} \in(0,1)$ and $\hat{F} \in[-1,1]$ hold true). For tractability of the stability analyses, I assume equal fitness of the homozygotes, $\mathrm{W}_{\mathrm{AA}}=\mathrm{W}_{\mathrm{aa}}=\mathrm{W}_{G}$, which simplifies the equilibrium so that $\hat{q}=1 / 2$ and $\hat{F}=\frac{1+(1-S) \mathrm{W}_{G}-\sqrt{(1+S)^{2} \mathrm{~W}_{G}^{2}+(2-6 S) \mathrm{W}_{G}+1}}{2\left(1-\mathrm{W}_{G}\right)} ;(\hat{q}, \hat{F})$ is internally stable $(0<\hat{q}<1)$ for any rate of partial selfing and any intensity of selection against 
166 homozygotes (Supplemental file). Stability under full selfing requires a restrictive

167 condition, namely a greater than two-fold heterozygote advantage (Supplemental file).

168 Treatment of modifier invasion under full selfing is postponed for a later section (Result

169

3).

170

171

172

173

174

175

176

177

178

179

180

181

182

183

184

185

186
The stability of $(\hat{q}, \hat{F})$ to the introduction of infinitesimal variation at $B$ (external stability)

can be ascertained by evaluating the Jacobian at equilibrium, which yields an upper

triangular block matrix, $\left.\mathbf{J}\right|_{(\widehat{\boldsymbol{q}}, \widehat{\boldsymbol{F}})}$. The submatrices on the diagonal are $\mathbf{J}_{\text {res }}$, a $3 \times 3$ matrix

corresponding to a resident population variable only at $A$, and $\mathbf{J}_{\text {mut }}$, a $7 \times 7$ matrix

corresponding to the dynamics of a rare mutant at $B$ (Supplemental file). The eigenvalues

of $\mathbf{J}_{\text {res }}$ are less than one in magnitude, and so stability is determined by the leading

eigenvalue $\left(\lambda_{L}\right)$ of $\mathbf{J}_{\text {mut }}$; if $\left|\lambda_{L}\right|>1$, then a rare $b$ allele invades at a geometric rate (Otto and

Day 2007). Assuming a resident population characterized by Mendelian ratios (i.e.

$\left.k_{m 0}=k_{f 0}=\frac{1}{2}\right)$

$\lambda_{L}=\frac{S\left(k_{f}\left(2-4 k_{m}\right)+2 k_{m}+\mathrm{W}_{G}-1\right)+\frac{1}{2} \sqrt{4\left(S\left(k_{f}\left(2-4 k_{m}\right)+2 k_{m}+\mathrm{W}_{G}-1\right)+\mathrm{W}_{G}+1\right)^{2}+64 S \mathrm{~W}_{G}\left(k_{f}\left(2 k_{m}-1\right)-k_{m}\right)}+\mathrm{W}_{G}+1}{S \mathrm{~W}_{G}+\sqrt{\mathrm{W}_{G}\left(S\left((S+2) \mathrm{W}_{G}-6\right)+\mathrm{W}_{G}+2\right)+1}+\mathrm{W}_{G}+1}$.

Result 1: The parameters for which $\left|\lambda_{L}\right|>1$ holds true are broad. Resident

Mendelian populations with partial selfing $(0<\mathrm{S}<1)$ and heterozygote advantage $\left(0 \leq \mathrm{W}_{G}\right.$

$<1)$ select for modifiers that impose oppositely-directed sex-specific meiotic drive of any

intensity:

or

$$
1 / 2<k_{m} \leq 1 \wedge 0 \leq k_{f}<1 / 2 .
$$


187 While the invasion analysis provides the conditions for the long-term spread of a rare modifier, it is of interest whether the time course of modifier evolution is appreciable with respect to the lifespan of a balanced polymorphism. Overdominance in the deterministic model results in a permanent stable equilibrium, but in nature, balanced polymorphisms only last as long they are not replaced by fitter genotypes in the course of environmental

192 changes or evolution of the genetic background (e.g. the resolution of overdominance by 193 gene duplication; Spofford 1969). Therefore, determining whether modifier evolution 194 occurs on a reasonable timescale is a necessary prerequisite to evaluating the importance 195 of the adaptive meiotic drive phenotype. Below, I numerically iterate cases of evolution over a time span of $10^{5}$ generations for a sample of parameters.

Numerical iterations of the two-locus model with strong magnitudes of drive and selection (assuming equal fitness of homozygotes and perfectly sex-reflected segregation schemes, $k_{m}=1-k_{f}$ ) are illustrated in Fig.1 and demonstrate fairly broad invasibility of driveenhancing modifiers starting from a frequency of $0.01 \%$ rising to "fixation" (> 99\%) within

$20210^{5}$ generations, which often occurred except for the weakest case of selection and drive depicted (i.e. $60: 40$ drive with $1 \%$ fitness reduction). A low selfing rate ( $\mathrm{S}=2.5 \%$ ) is seen to

204 be uniformly associated with the slowest rate of evolution; multiple cases of modifier

205 fixation are observed for low selfing nonetheless. As is intuitive, higher intensities of 206 distortion and stronger selection against homozygotes are each associated with quicker 207 fixation of the drive enhancer. 
208 The lack of appreciable modifier evolution within $10^{5}$ generations for the weakest

209 intensities of drive and selection in Fig. 1 calls for an investigation into cases where only

210 one of these processes exhibits small values, while the other process is strong. An

211 examination of cases reveals that weak segregation distortion and strong selection does

212 not guarantee timely evolution of modifiers (Fig. 2), nor does weak selection and strong

213 distortion (Fig. 3). Nevertheless, with coefficients of sufficient magnitude, cases of drive

214 enhancer fixation are apparent for various levels of selfing. It should be noted that in

215 general, the relation between the selfing rate and the pace of modifier evolution is non-

216 monotonic; an expression for the selfing parameter that maximizes the rate of modifier

217 invasion can be derived analytically, in terms of $\mathrm{W}_{\mathrm{G}}$ and $k$, where $k=k_{m}=1-k_{f}$

218 (Supplemental file, Fig. S1).

219 The invasion analysis (Result 1) and the preceding numerical results took on the

220 restrictive assumption that homozygote fitnesses were equal, and so I also present a

221 sample of numerical results where this assumption is relaxed. A difference in fitness

222 between the homozygotes (AA and aa) of course results in lower initial heterozygosity at $A$,

223 and a slower invasion rate of the modifier as compared to the case of equal homozygote

224 fitness (Fig. 4a,b); a shift in the frequency at the $A$ locus occurs away from its Mendelian

225 equilibrium to a new value. In addition, if asymmetrically sex-reflected ratios (i.e. $k_{m} \neq 1$ -

$226 k_{f}$ ) are investigated, a modifier polymorphism can appear (Fig. 4c-d). In some cases (Fig.

227 4c), the mutant modifier winds up at an internal neutral equilibrium because the process of

228 modifier selection causes a frequency shift at the $A$ locus into a fixation state; such an event

229 stops any further directional selection at the $B$ locus. In other cases (Fig. 4d), alleles at $A$

230 and $B$ are both internally stabilized; for the $B$ locus, this owes to a balance of forces 
231 between (1) an adaptive reduction in the segregation load (i.e. lowered homozygote

232 production) and (2) a maladaptive imposition of a drive load caused by the frequency shift

233 at A (Úbeda and Haig 2005). Yet other cases of asymmetric drive cause a shift at $A$ but still

234 allow for the modifier mutant to fix (Fig. 4e,f).

External stability of all-and-none to drive suppressors in a partial selfing population

236 Úbeda and Haig (2005) reported that all-and-none segregation is stable to mutants of the

237 segregation scheme in random mating populations. I investigate whether all-and-none

238 exhibits stability under partial selfing. The dynamical equations now assume $\mathrm{k}_{\mathrm{m} 0}=1$ and $\mathrm{k}_{\mathrm{f} 0}$

$239=0$ (i.e. one of the two possible all-and-none phenotypes; $\mathrm{k}_{\mathrm{m} 0}=0$ and $\mathrm{k}_{\mathrm{f} 0}=1$ would do just

240 as well). With equal homozygote fitnesses, the single-locus equilibrium associated with all-

241 and-none segregation, selfing, and overdominance can be shown to equal $\hat{q}=\frac{1}{2}, \hat{F}=-1$,

242 which is an internally stable equilibrium consisting entirely of AaBB genotypes

243 (Supplemental file). The eigenvalues of $\mathbf{J}_{\text {mut }}$ in this case are:

$$
\lambda_{1}=0
$$

$\lambda_{2}=\frac{1}{2} S\left(k_{f}\left(1-2 k_{m}\right)+k_{m}\right)$

$\lambda_{3}=\lambda_{4}=\frac{s \mathrm{~W}_{G}}{2}$,

$$
\lambda_{5}=\frac{1}{2}(S+1) \mathrm{W}_{G}
$$

$$
\lambda_{6}=\frac{1}{4}\left(S\left(k_{f}\left(1-4 k_{m}\right)+3 k_{m}+\mathrm{W}_{\mathrm{G}}-1\right)-\right.
$$




$$
\lambda_{7}=\frac{1}{4}\left(S\left(k_{f}\left(1-4 k_{m}\right)+3 k_{m}+\mathrm{W}_{\mathrm{G}}-1\right)+\right.
$$
$\sqrt{\left(k_{f}\left(1-4 S k_{m}+S\right)+(3 S-1) k_{m}+S\left(\mathrm{~W}_{\mathrm{G}}-1\right)+\mathrm{W}_{\mathrm{G}}+1\right)^{2}+8 \mathrm{~W}_{\mathrm{G}} k_{m}\left(4 S k_{f}-3 S+1\right)-8(S+1) \mathrm{W}_{\mathrm{G}} k_{f}}+$

Result 2: None of the eigenvalues of $\mathbf{J}_{\text {mut }}$ for an all-and-none resident phenotype (Eq. 5a-f) are ever greater than one in magnitude in a partial selfing population. $\lambda_{7}$ takes the unit value when the mode of all-and-none segregation mutates from $\left(k_{m 0}=1, k_{f 0}=0\right)$ to $\left(k_{m}=\right.$ $\left.0, k_{f}=1\right)$. Otherwise, all eigenvalues are less than one. This demonstrates the long-term stability of all-and-none segregation to any mutants of the segregation scheme in a partial selfing population with symmetric overdominance and equal fitness of homozygotes.

As an extension of Result 2, it can be shown that the direct invasion of Mendelian segregation $\left(k_{m}=1 / 2, k_{f}=1 / 2\right)$ into a resident all-and-none population cannot occur for generalized values of $\mathrm{W}_{\mathrm{AA}}$ and $\mathrm{W}_{\mathrm{aa}}$ (i.e. with no constraint on their equality); the eigenvalues are complicated (Supplemental file). Similarly, the direct invasion of a sex-

264 limited Mendelian ratio (and maintenance of maximal distortion in the other sex) is also 265 selected against in these more general circumstances (Supplemental file). equilibrium can be readily derived for any initial genotype frequencies and general parameters. Following Karlin (1968), given a 10x10 matrix (T) that transforms the row

270 vector of two-locus diploid genotype frequencies (p) on a generation-by-generation basis,

271 the time course of evolution can be written as: $\mathbf{p}^{(n)}=\frac{\mathbf{p}^{(\mathbf{0})} \mathbf{T}^{n}}{\prod_{i=0}^{n-1} \kappa_{i}}$, where $n$ is the number of 
272

273

274

275

276

277

278

279

287 for the generalized case. inner product.

generations, $\kappa$ is a normalizing constant that ensures the elements of $\mathbf{p}$ add up to one in each generation, and $\mathbf{p}^{(\mathbf{0})}$ is the vector of initial values. As $n \rightarrow \infty$, the vector $\mathbf{p}^{(n)}$ converges to the equilibrium vector $\mathbf{M}$. If an adaptive drive-enhancing modifier is introduced into a population at stable equilibrium owing to overdominance (i.e. $0 \leq \mathrm{W}_{A A}$, $\mathrm{W}_{a a}<1 / 2$ ), then the leading eigenvalue of $\mathbf{T}$ is $\lambda=k_{f}+k_{m}-2 k_{f} k_{m}$, which has multiplicity 1 . The vector $\mathbf{p}^{(n)}$ as $n \rightarrow \infty$ can be written as $\mathbf{M}=\frac{\gamma\left(\boldsymbol{p}^{(\mathbf{0})}, \delta\right)}{\sum \gamma\left(\boldsymbol{p}^{(\mathbf{0})}, \delta\right)}$, where $\gamma$ and $\delta$ are respectively the left and right eigenvectors associated with $\lambda$, normalized to be biorthogonal, and $\left(\boldsymbol{p}^{(0)}, \delta\right)$ is the

Result 3: Under full selfing, the equilibrium two-locus genotype frequencies are

$$
\hat{p}_{22}=-\left(\frac{\left(1-k_{f}\right)\left(1-k_{m}\right)\left(k_{f}\left(2 k_{m}-1\right)-k_{m}+W_{a a}\right)}{k_{f} k_{m}\left(W_{a a}+W_{A A}-2\right)-k_{f}\left(W_{A A}\right)+k_{f}-\left(W_{A A}-1\right)\left(k_{m}-W_{a a}\right)}\right),
$$

, which correspond to positive frequencies for only the $\mathrm{Ab} / \mathrm{Ab}, \mathrm{Ab} / \mathrm{ab}$, and $\mathrm{ab} / \mathrm{ab}$ genotypes, indicating globally convergent fixation of an adaptive drive-enhancing modifier

$$
\hat{p}_{11}, \hat{p}_{12}, \hat{p}_{13}, \hat{p}_{14}, \hat{p}_{23}, \hat{p}_{33}, \hat{p}_{34}=0
$$

All invading adaptive drive-enhancers converge to fixation, even for asymmetricallyreflected drive schemes, and so there are no conditions for stable or neutral modifier polymorphisms as under partial selfing. With the establishment of sufficient distortion, 
equilibria are characterized by negative inbreeding coefficients owing to an excess of

292

293

294

295

296

297

298

299

300

301

302

303

304

305

306

307

308

309

310

311

312

heterozygotes, relative to panmictic Hardy-Weinberg proportions, even though selfing is

maximal (Fig. 5). Upon drive-enhancer fixation, the only modifiers which can subsequently

invade are those that impose even stronger oppositely-directed distortion. All-and-none

segregation under full selfing and stable heterozygote advantage is therefore expected to persist over long-term evolution.

\section{Discussion}

The evolutionary instability of the Mendelian scheme on theoretical grounds is seen to owe to the tendency of overdominance to select for sex-specific segregation-distorting

phenotypes. While earlier work found that adaptive drive under symmetric overdominance is selected for at a slow arithmetic rate in panmictic populations (the leading eigenvalue corresponding to modifier invasion being at most 1 in magnitude; see Úbeda and Haig 2005, where second-order terms of a Hessian matrix were analyzed in order to ascertain invasion conditions), the inclusion of non-random mating in the model clearly transforms the pace of modifier invasion to that of geometric increase (modifier invasion with a leading eigenvalue greater than 1; Result 1, present article). Under panmixia, two mutant individuals would need to mate with each other in order to produce a super-Mendelian proportion of heterozygous progeny, but under mixed or obligate selfing, overproduction of heterozygotes is an immediate consequence of a single mutant individual. In other words, modifier effects on the fitness of selfers are first order since the expression of a fitness advantage does not depend on mating encounters between rare mutants. The relation between inbreeding and the evolution of adaptive drive also stands in stark 
contrast to the suppressive effect of inbreeding on the spread of selfish drivers (Burt and Trivers 1999, Bull 2017).

The interaction of selfing and all-and-none segregation in the present work is consonant with Charlesworth's (1979) model investigating the spread of sex-limited gamete lethals (or detrimentals) in a system of selfing and balanced homozygotic lethality, characteristics which are common in species with permanent translocation heterozygosity. Charlesworth found that the invasion of a sex-limited gamete lethal (in sperm, say) is effectively neutral under full selfing, but disadvantageous at lower selfing rates; subsequent invasion of an opposite-sex gamete lethal (i.e. lethal eggs of the other allelic type) is selectively favored since this would avoid production of lethal homozygous progeny; the all-and-none scheme could thus become fixed. Holsinger and Ellstrand (1984) remark that an even less restrictive picture would emerge if the gamete lethals of Charlesworth's model were replaced with meiotic drivers.

Following Eshel (1985) and Úbeda and Haig (2005), here I adopt the modifier's eye view of long-term evolution, rather than focusing on the short-term dynamics of meiotic drive alleles per se. The modifier perspective is key, since whatever the short-term evolutionary dynamics of any particular driver may be, the ultimate fate of drivers over the long-term will owe to the make-up of the genetic background (Eshel 1996, Eshel and Feldman 2001). If the genes capable of modifying drive phenotypes overwhelmingly benefit from suppressing such behavior, then any particular meiotic driver is destined for extinction on a timescale that is limited only by the introduction and spread of suppressor alleles at the relevant loci. Given that unlinked modifiers are likely to be more numerous than linked 
modifiers from the perspective of any particular distorter, the dissent or approval of the permanent unlinked majority of the parliament of genes is decisive for determining the long run stability of the drive phenotype. With non-zero rates of self-fertilization, the unlinked majority ought to favor the repeal of Mendelian law at any locus that is at a stable equilibrium due to classical heterozygote advantage. Under random mating, this expectation holds for asymmetric overdominance, but only mild assent is expected for the classical symmetric case (Úbeda and Haig 2005). It remains to be discovered whether there exist other classes of initial population conditions that effect the invasion of unlinked driveenhancers.

Given the ubiquity of Mendelian segregation in nature, how come real populations do not conform to theoretical expectation? One possibility is suggested in the numerical results, which indicate that a concatenation of weak coefficients in the selection and segregation processes fails to effect the swift establishment of a mutant modifier. The lifespan of a balanced fitness polymorphism that is the target of adaptive drive presumably sets a time limit during which the appropriate modifier mutations must originate, survive genetic drift at the extinction boundary, and then rise from rarity to an appreciable frequency. If typical balanced polymorphisms do not last long enough for the full course of evolutionary changes needed for the establishment of such modifiers, then the ubiquity of Mendelian segregation follows. In allowing for the occasional case of long-persisting overdominance, such a constraint permits the odd example of adaptive drive as in the permanent translocation heterozygotes of Oenothera that adhere to the all-and-none scheme in gametogenesis (Cleland 1972, Holsinger and Ellstrand 1984, Harte 1994). A related issue is that heterozygote advantage might often be associated with rapid allelic turnover (Sellis et 

While these considerations and others may be relevant, at present a decisive argument for

al. 2011) in a manner that interferes with an orderly process of modifier selection. Furthermore, as some of the numerical examples of asymmetrically-reflected drive ratios reveal, some mutants can knock out the polymorphism of heterotic alleles, and in doing so collapse the basis for their own stable persistence.

As mentioned by Úbeda and Haig (2005), male fertility costs associated with segregation distorters can also be a serious constraint (Haig and Bergstrom 1995). For selfers, the costs of reduced male fertility under adaptive drive would strongly depend on the intensity of inter-individual male gamete competition. Such competition is presumably less intense for predominant selfers, assuming a negative relationship between the rate of selfing and population density (Karron et al. 1995, Morgan, Wilson and Knight 2005). Populations with intermediate and low selfing rates, however, would indeed seem to be severely affected by this constraint.

Additionally, there is a lack of realism associated with having a single modifier directly control the production of both male and female gametes (Úbeda 2006). An attempt to adhere closely to realism would involve investigating a rather complicated model that incorporates two sex-limited modifier genes encoded at different loci (one for each sex), as well as multiple alleles at the fitness locus representing the simultaneous variation at $A$ of sex-limited distorters and wild-type Mendelian alleles. It is not clear whether the simplifying assumption on modifier control is ultimately misleading. why all-and-none segregation is not the normal mode of inheritance in natural populations, selfing or otherwise, has not been established. The near-universality of Mendelian 
segregation rests securely on observation but lacks a convincing adaptationist explanation.

Perhaps a conclusive idea of the pertinent constraints will emerge from more realistic multi-locus scenarios. What is clear is that our understanding has shifted from the feeling of a satisfying denouement derived from earlier sex-independent models to a sort of puzzlement that stems from consideration of sex-differentiated meiosis.

\section{Data accessibility}

A Mathematica notebook (Wolfram Research, Inc. 2018, ver 11.2) and an R script (R Core Team 2018) for numerical iteration are available at: https://github.com/ebrud/adaptive_meiotic_drive.

Acknowledgements: I thank Walter Eanes, Mark Kirkpatrick, John True, and Joshua Rest for helpful comments on the manuscript.

\section{References}

Bull, James J. "Lethal gene drive selects inbreeding." Evolution, medicine, and public health 2017, no. 1 (2017): 1-16.

Brandvain, Yaniv, and Graham Coop. "Sperm should evolve to make female meiosis fair." Evolution 69, no. 4 (2015): 1004-1014.

Burt, Austin, and Robert Trivers. "Selfish DNA and breeding system in flowering plants." Proceedings of the Royal Society of London. Series B: Biological Sciences 265, no. 1391 (1998): 141-146.

Burt, Austin and Robert Trivers. Genes in conflict: the biology of selfish genetic elements. Harvard University Press, 2006.

Charlesworth, Brian. "Selection for gamete lethals and S-alleles in complex heterozygotes." Heredity 43, no. 2 (1979): 159-164.

Cleland, Ralph E. Oenothera: Cytogenetics and Evolution. Academic Press, 1972.

Crow, James F. "Why is Mendelian segregation so exact?" BioEssays 13, no. 6 (1991): 305312.

Didion, John P., Andrew P. Morgan, Liran Yadgary, Timothy A. Bell, Rachel C. McMullan, Lydia Ortiz de Solorzano, Janice Britton-Davidian et al. "R2d2 drives selfish sweeps in the house mouse." Molecular biology and evolution 33, no. 6 (2016): 1381-1395. 
Dunn, L. C. "Evidence of evolutionary forces leading to the spread of lethal genes in wild populations of house mice." Proceedings of the National Academy of Sciences of the United States of America 43, no. 1 (1957): 158-163.

Eshel, Ilan. "Evolutionary genetic stability of Mendelian segregation and the role of free recombination in the chromosomal system." The American Naturalist 125, no. 3 (1985): 412-420.

Eshel, Ilan. "On the changing concept of evolutionary population stability as a reflection of a changing point of view in the quantitative theory of evolution." Journal of mathematical biology 34, no. 5-6 (1996): 485-510.

Eshel, Ilan, and Marcus W. Feldman. "Optimality and evolutionary stability under shortterm and long-term selection." Adaptationism and Optimality (ed. Steven Orzack and Elliot Sober). Cambridge University Press, 2001.

Fishman, Lila, and Arpiar Saunders. "Centromere-associated female meiotic drive entails male fitness costs in monkeyflowers." Science 322, no. 5907 (2008): 1559-1562.

Gershenson, S. "A new sex-ratio abnormality in Drosophila obscura." Genetics 13, no. 6 (1928): 488-507.

Haig, David, and Carl T. Bergstrom. "Multiple mating, sperm competition and meiotic drive." Journal of Evolutionary Biology 8, no. 3 (1995): 265-282.

Harte, Cornelia. Oenothera: contributions of a plant to biology. Springer, 1994.

Hartl, Daniel L. "Modifier theory and meiotic drive." Theoretical population biology 7, no. 2 (1975): 168-174.

Hayman, B. I. "Mixed selfing and random mating when homozygotes are at a disadvantage." Heredity 7, no. 2 (1953): 185-192.

Holsinger, Kent E., and Norman C. Ellstrand. "The evolution and ecology of permanent translocation heterozygotes." The American Naturalist 124, no. 1 (1984): 48-71.

Karlin, Samuel. "Equilibrium behavior of population genetic models with non-random mating." Journal of Applied Probability 5, no. 2 (1968): 231-313.

Karron, Jeffrey D., Nina N. Thumser, Rosella Tucker, and Amy J. Hessenauer. "The influence of population density on outcrossing rates in Mimulus ringens." Heredity 75, no. 2 (1995): 175-180.

Liberman, Uri. "Modifier theory of meiotic drive: is Mendelian segregation stable?" Theoretical population biology 10, no. 2 (1976): 127-132.

Liberman, Uri. "Mendelian segregation: a choice between "order" and "chaos"." Journal of mathematical biology 28, no. 4 (1990): 435-449. 
Liberman, Uri, and Marcus W. Feldman. "On the evolutionary significance of Mendel's ratios." Theoretical population biology 17, no. 1 (1980): 1-15.

Liberman, Uri, and Marcus W. Feldman. "On the evolution of fluctuating segregation distortion." Theoretical Population Biology 21, no. 3 (1982): 301-317.

Leigh, Egbert G. "How does selection reconcile individual advantage with the good of the group?" Proceedings of the National Academy of Sciences 74, no. 10 (1977): 45424546.

Lloyd, David G. "Gene selection of Mendel's rules." Heredity 53, no. 3 (1984): 613-624.

Morgan, Martin T., William G. Wilson, and Tiffany M. Knight. "Plant population dynamics, pollinator foraging, and the selection of self-fertilization." The American Naturalist 166, no. 2 (2005): 169-183.

Nuckolls, Nicole L., María Angélica Bravo Núñez, Michael T. Eickbush, Janet M. Young, Jeffrey J. Lange, Jonathan S. Yu, Gerald R. Smith, Sue L. Jaspersen, Harmit S. Malik, and Sarah E. Zanders. "wtf genes are prolific dual poison-antidote meiotic drivers." eLife 6 (2017).

Otto, Sarah P., and Troy Day. A biologist's guide to mathematical modeling in ecology and evolution. Princeton University Press, 2007.

R Core Team. "R: A language and environment for statistical computing." R Foundation for Statistical Computing, Vienna, Austria. (2018) https://www.R-project.org/.

Rhoades, M. M. "Preferential segregation in maize." Genetics 27, no. 4 (1942): 395-407.

Sandler, L., Yuichiro Hiraizumi, and Iris Sandler. "Meiotic drive in natural populations of Drosophila melanogaster. I. The cytogenetic basis of segregation-distortion." Genetics 44, no. 2 (1959): 233-250.

Sellis, Diamantis, Benjamin J. Callahan, Dmitri A. Petrov, and Philipp W. Messer. "Heterozygote advantage as a natural consequence of adaptation in diploids." Proceedings of the National Academy of Sciences 108, no. 51 (2011): 20666-20671.

Spofford, Janice B. "Heterosis and the evolution of duplications." The American Naturalist 103, no. 932 (1969): 407-432.

Thomson, Glenys J., and Marcus W. Feldman. "Population genetics of modifiers of meiotic drive III. Equilibrium analysis of a general model for the genetic control of segregation distortion." Theoretical population biology 10, no. 1 (1976): 10-25.

Úbeda, Francisco, and David Haig. "Sex-specific meiotic drive and selection at an imprinted locus." Genetics 167, no. 4 (2004): 2083-2095. 
479 Úbeda, Francisco, and David Haig. "On the evolutionary stability of Mendelian segregation." Genetics 170, no. 3 (2005): 1345-1357.

481 Úbeda, Francisco. "Why Mendelian segregation?" Biochemical Society Transactions 34, no.4 482 (2006): 566-568.

483 Wolfram Research, Inc., Mathematica, Version 11.3, Champaign, IL (2018).

484 Workman, P. L., and S. K. Jain. "Zygotic selection under mixed random mating and self- 


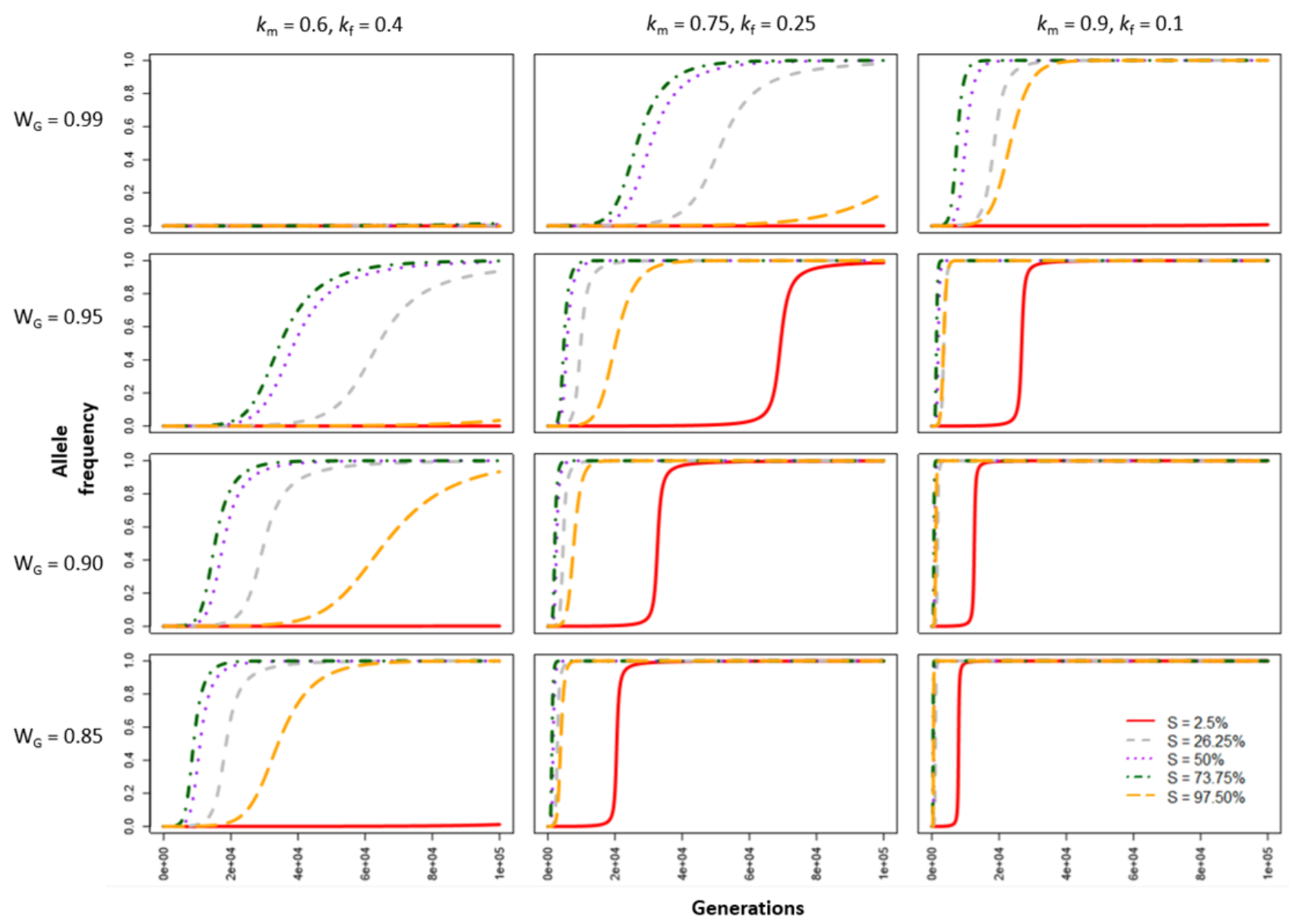

Fig 1. Evolutionary trajectories of the $b$ allele for a sample of 'strong' parameter combinations. Evolution in these trajectories is depicted over $10^{5}$ generations, starting from an initial frequency of $10^{-4}$ for the modifier mutant and linkage equilibrium with the fitness locus $A$. Alleles at the $A$ locus remain at a frequency of $50 \%$ throughout due to symmetry of the parameter values. For a particular panel, homozygous fitness values are given by the row label and segregation ratios by the column label. 

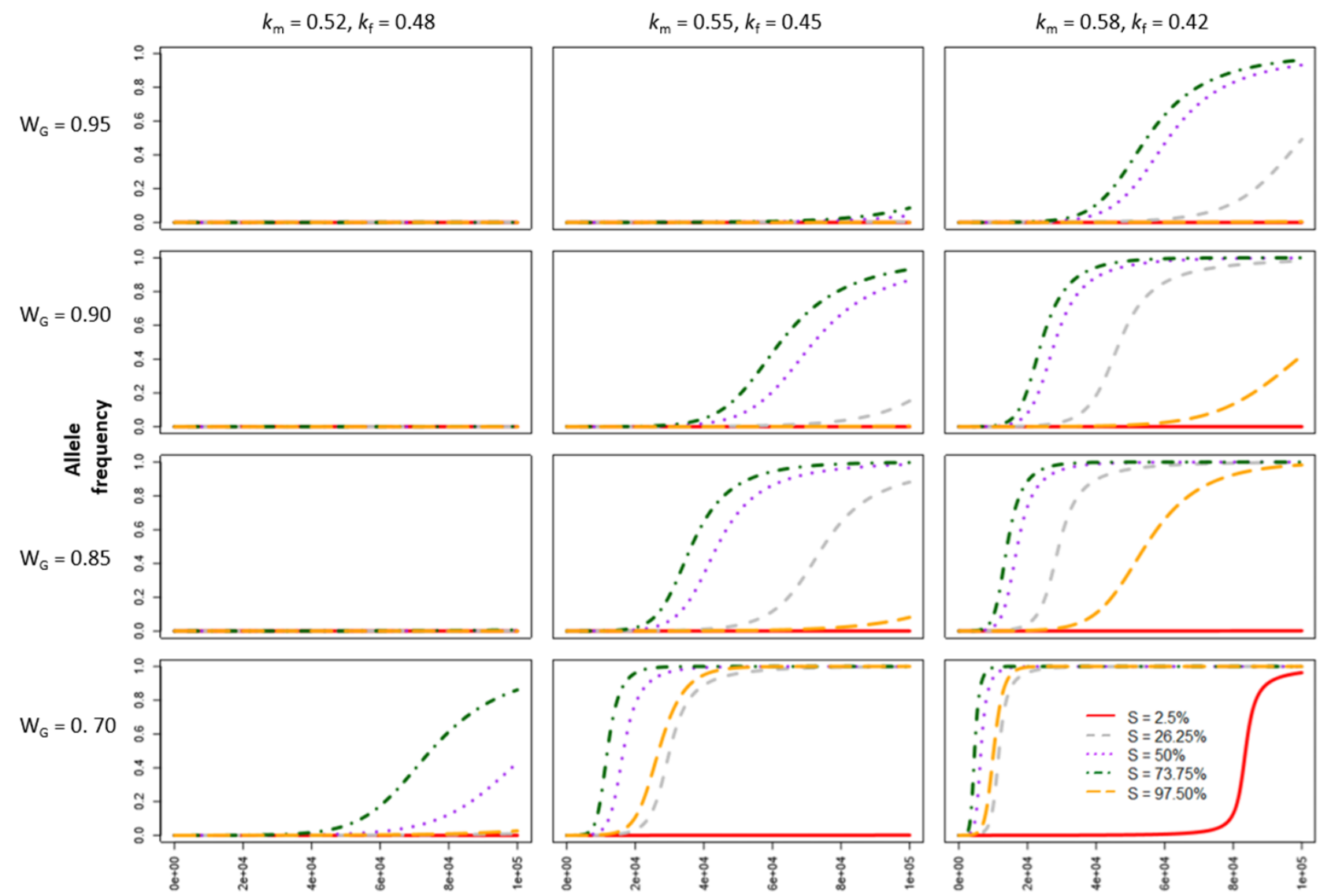

Fig 2. Evolutionary trajectories of the $b$ allele for a sample of parameters with strong selection and 'weak' segregation distortion. Evolution in these trajectories is depicted over $10^{5}$ generations, starting from an initial frequency of $10^{-4}$ for the modifier mutant and linkage equilibrium with the fitness locus $A$. Alleles at the $A$ locus remain at a frequency of $50 \%$ throughout due to symmetry of the parameter values. For a particular panel, homozygous fitness values are given by the row label and segregation ratios by the column label. 


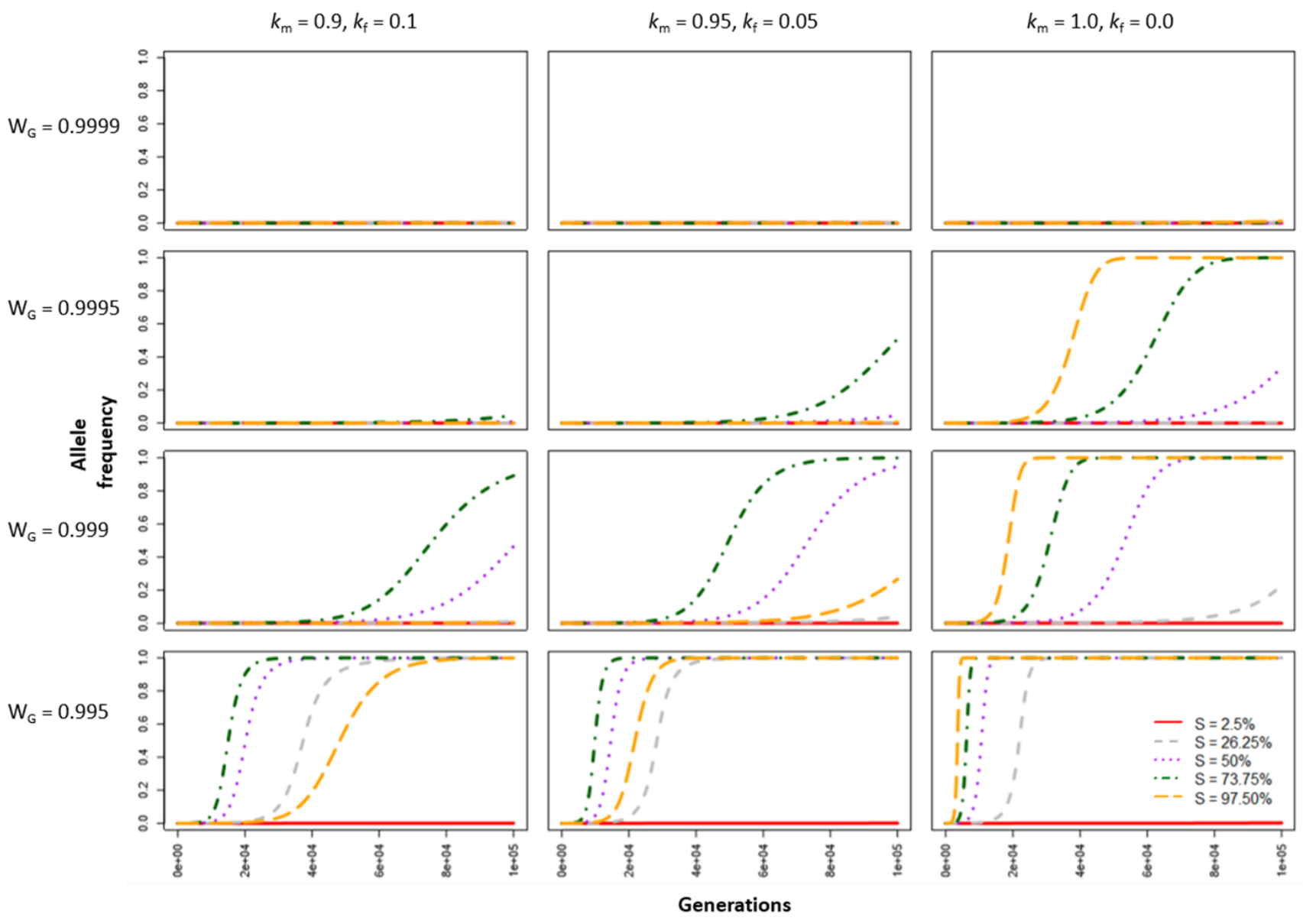
selection and strong segregation distortion. Evolution in these trajectories is depicted over $10^{5}$ generations, starting from an initial frequency of $10^{-4}$ for the modifier mutant and linkage equilibrium with the fitness locus $A$. Alleles at the $A$ locus remain at a frequency of $50 \%$ throughout due to symmetry of the parameter values. For a particular panel, homozygous fitness values are given by the row label and segregation ratios by the column label. 

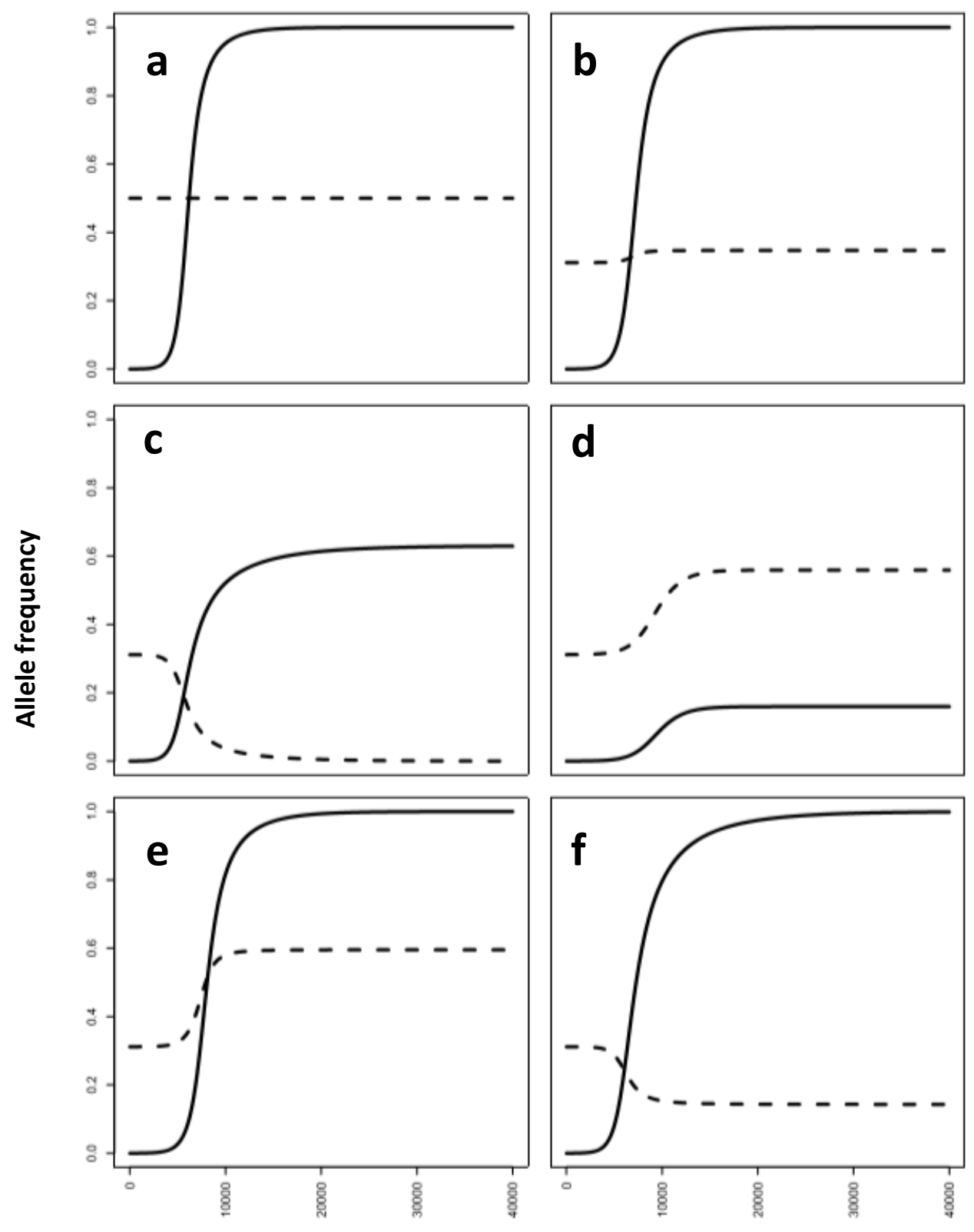

Generations

578 homozygous fitnesses and segregation schemes. (Panels a-f) Modifier " $b$ " allele (solid

579 line) is introduced into a Mendelian population at a frequency of $10^{-4}$ and the "a" allele

580 (dashed) starts at the deterministic equilibrium for single-locus heterosis under equal segregation; change over 40,000 generations is displayed. $S=0.37$ and $k_{m}=0.77$ (i.e. $k_{m}=1-k_{f}$ ): $\mathrm{W}_{\mathrm{AA}}=\mathrm{W}_{\mathrm{aa}}=0.95, k_{f}=0.23$. (Panels b-f) Asymmetric homozygous fitnesses: $\mathrm{W}_{\mathrm{AA}}=0.962, \mathrm{~W}_{\mathrm{aa}}=0.938$. (b) $k_{f}=0.23$. (c) $k_{f}=0.19$. (d) $k_{f}=0.315$. (e) $k_{f}=0.255$. (f) $k_{f}=$ 0.21 . 


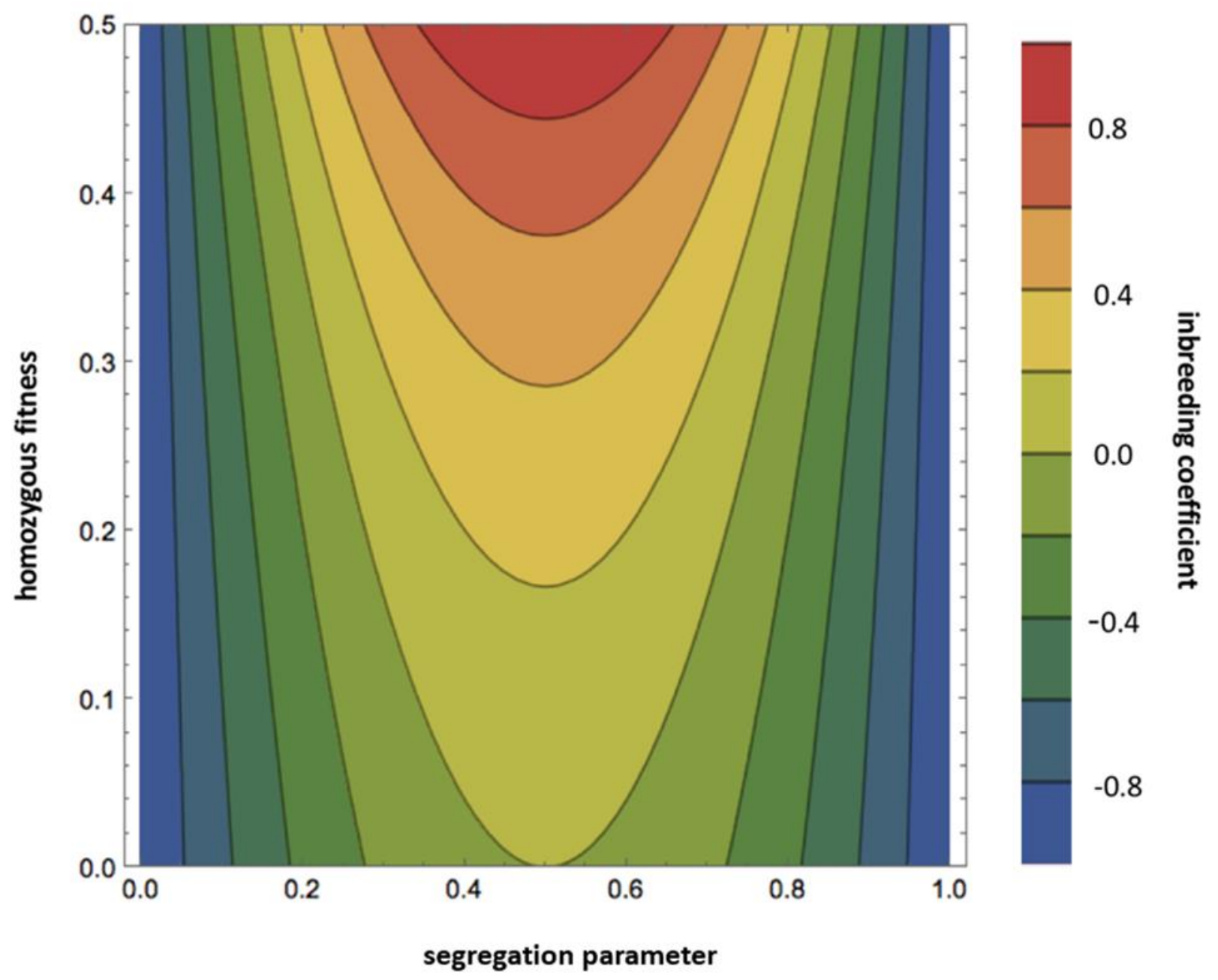
disadvantage, perfectly sex-reflected segregation ratios $\left(k_{m}=1-k_{f}\right)$, and obligate selfing. Contours demarcate changes in $\hat{F}$ at intervals of 0.2 . The parameter space includes only homozygous fitnesses of $<50 \%$. Areas of darker green and blue correspond to negative inbreeding coefficients at equilibrium: $\hat{F}<0$ whenever $\mathrm{W}_{\mathrm{G}}<(1-2 k)^{2}$. Due to parameter symmetries assumed in this plot, $\hat{q}=0.5$ throughout. 


\section{Appendix}

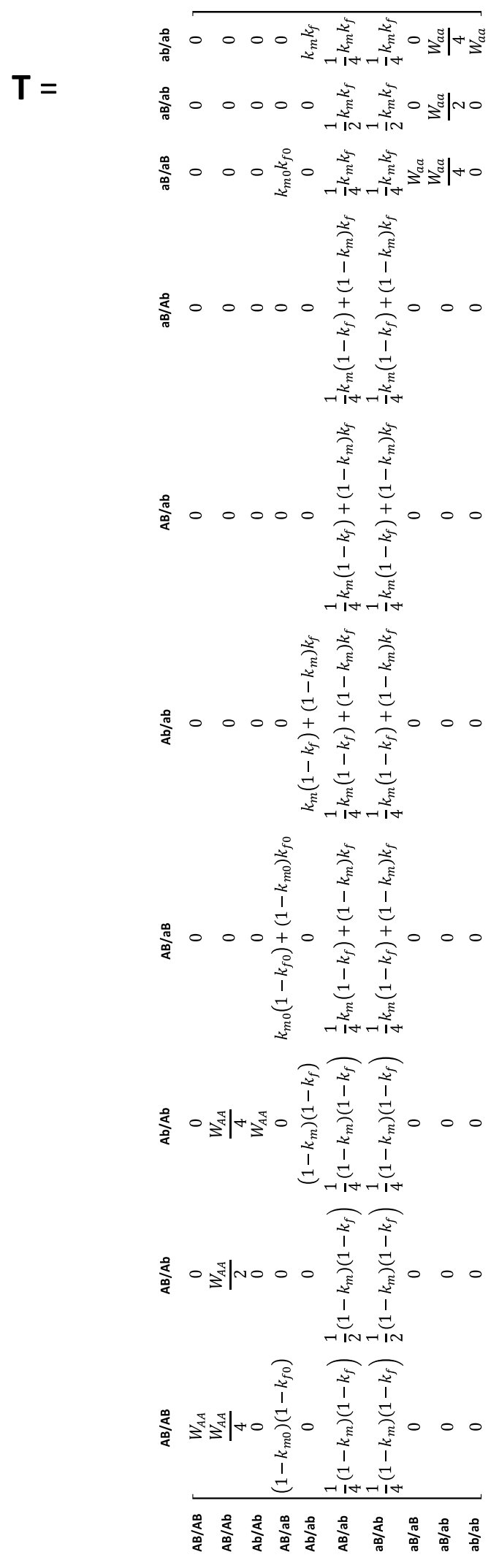

Explanation of the T matrix: the parent genotype indicated on the row label produces the offspring indicated on the column labels, according to the proportions given by the elements. The elements incorporate (1) selection, (2) free recombination, and (3) arbitrary segregation parameters. The matrix is comparable to that of Karlin (1968, Table 4 , p. 246), who assumed equal segregation and arbitrary recombination.

(The T matrix describes the production of offspring due to selfing. If random mating also occurs (i.e. mixed mating, $0<\mathrm{S}<1$ ), then the $\mathbf{U}$ and $\mathbf{V}$ matrices describing outcrossing are also involved. See the description of the Model in the main text).

Explanation of the $\mathbf{U}$ and $\mathbf{V}$ matrices: the genotypes on the row labels produce the gametes indicated on the column labels in the proportions given by the elements, incorporating processes 1-3 as in the paragraph above. See next page.

While the $\mathbf{T}$ matrix directly figures in an analytical derivation (see the section on obligate selfing), the $\mathbf{U}$ and $\mathbf{V}$ matrices are merely convenient references for the compact representation of the system of equations given in the Model section. 
602

$603 \mathbf{U}=$

604

\begin{tabular}{|c|c|c|c|c|}
\hline & AB & $\mathbf{A b}$ & aB & $a b$ \\
\hline$A B / A B$ & $W_{A A}$ & 0 & 0 & 0 \\
\hline$A B / A b$ & $\frac{W_{A A}}{?}$ & $\frac{W_{A A}}{?}$ & 0 & 0 \\
\hline$A b / A b$ & $\begin{array}{l}2 \\
0\end{array}$ & ${\stackrel{2}{W_{A A}}}$ & 0 & 0 \\
\hline$A B / a B$ & $1-k_{m 0}$ & 0 & $k_{m 0}$ & 0 \\
\hline$A b / a b$ & 0 & $1-k_{m}$ & 0 & $k_{m}$ \\
\hline$A B / a b$ & $\underline{1-k_{m}}$ & $\underline{1-k_{m}}$ & $\frac{k_{m}}{n}$ & $\frac{k_{m}}{2}$ \\
\hline$A b / a B$ & $\begin{array}{c}2 \\
1-k_{m} \\
\end{array}$ & $\begin{array}{c}2 \\
1-k_{m} \\
\end{array}$ & $\begin{array}{c}2 \\
k_{m} \\
\end{array}$ & $\begin{array}{c}2 \\
k_{m} \\
\end{array}$ \\
\hline $\mathrm{aB} / \mathrm{aB}$ & $\begin{array}{l}2 \\
0\end{array}$ & $\begin{array}{l}2 \\
0\end{array}$ & $\begin{array}{c}\overline{2} \\
W_{a a}\end{array}$ & $\begin{array}{l}2 \\
0\end{array}$ \\
\hline$a B / a b$ & 0 & 0 & $\frac{W_{a a}}{2}$ & $\frac{W_{a a}}{2}$ \\
\hline$a b / a b$ & 0 & 0 & $\begin{array}{l}2 \\
0\end{array}$ & $\left.\begin{array}{c}2 \\
W_{a a}\end{array}\right]$ \\
\hline
\end{tabular}

605

$606 \mathrm{~V}=$

\begin{tabular}{|c|c|c|c|c|}
\hline & $A B$ & $A b$ & $\mathrm{aB}$ & $a b$ \\
\hline$A B / A B$ & $W_{A A}$ & 0 & 0 & 0 \\
\hline$A B / A b$ & $\frac{W_{A A}}{2}$ & $\frac{W_{A A}}{2}$ & 0 & 0 \\
\hline$A b / A b$ & 0 & $W_{A A}$ & 0 & 0 \\
\hline$A B / a B$ & $1-k_{f 0}$ & 0 & $k_{f 0}$ & 0 \\
\hline $\mathrm{Ab} / \mathrm{ab}$ & $\begin{array}{c}0 \\
1-k_{f}\end{array}$ & $\begin{array}{l}1-k_{f} \\
1-k_{f}\end{array}$ & $\begin{array}{c}0 \\
k_{f}\end{array}$ & $\begin{array}{l}k_{f} \\
k_{f}\end{array}$ \\
\hline$A B / a b$ & $\frac{2}{1-k_{f}}$ & $\begin{array}{c}2 \\
1-k_{f}\end{array}$ & $\begin{array}{l}\overline{2} \\
k_{f}\end{array}$ & $\begin{array}{l}2 \\
k_{f} \\
\end{array}$ \\
\hline $\mathrm{Ab} / \mathrm{aB}$ & 2 & 2 & $\frac{1}{2}$ & $\frac{1}{2}$ \\
\hline$a B / a B$ & 0 & 0 & $W_{a a}$ & $\begin{array}{r}0 \\
\end{array}$ \\
\hline$a B / a b$ & 0 & 0 & $\frac{W_{a a}}{2}$ & $\frac{w_{a a}}{2}$ \\
\hline$a b / a b$ & 0 & 0 & 0 & $W_{a a}$ \\
\hline
\end{tabular}

